\title{
Deformation Mechanism of Calender Films and Melt Spun Fibers Produced by Low Molecular Weight Polyethylene
}

\author{
Seog Joo $\mathrm{KANG}^{\dagger}$ and Masaru MATSUO* \\ Department of Clothing Science, Faculty of Home Economics, \\ Nara Women's University, Nara 630, Japan
}

(Received April 11, 1988)

\begin{abstract}
Difference of the mechanical properties between calender films and melt spun fibers, produced by using low molecular weight polyethylene, was discussed in terms of morphological aspects. The viscosity-average molecular weights of the calender films and the melt spun fibers were 70,000 and 54,000 , respectively. The orientation factor of the $c$-axis of the film with a maximum draw ratio of 14 was 0.9291 , indicating a high orientational degree of the $c$-axis with respect to the machine direction. This value is slightly higher than that of the drawn fiber $(0.8423)$. In contrast, the Young's modulus of the drawn film was only $15.3 \mathrm{GPa}$, while the Young's modulus of the drawn fiber reached $55.6 \mathrm{GPa}$. The results were explained by the difference of the morphological properties which were obtained by using small angle $\mathrm{X}$-ray scattering, wide angle $\mathrm{X}$ ray diffraction, small angle light scattering, and birefringence measurements. A conclusion drawn from this work is as follows; the preferential orientation of the $c$-axes of the films is mainly due to the rotation of crystallites, while that for the fibers is due to the crystal transformation from a folded to a fibrous type.

KEY WORDS Low Molecular Weight Polyethylene / The Orientation Factor of the $c$-Axes / Young's Modulus / Rotation of Crystallites / Crystal Transformation /
\end{abstract}

It is well known that the theoretical Young's modulus and tensile strength of polymeric materials can only be realized if the chains are fully aligned and extended and if the specimen is almost completely crystalline. In an attempt to produce such an ideal sample, chain alignment induced by deformation or flow has been investigated extensively. On the basis of this concept, the preparation of polymeric fibersand films with high modulus has been studied and results of interest have been obtained for ultra-high molecular weight polyethylene (UHMWPE) whose molecular weight is higher than $1 \times 10^{6} .^{1-11} \mathrm{~A}$ question arises whether it is essentially impossible to produce such high modulus and high strength fibers by gelation/ crystallization from solutions using low molecular weight polyethylene (LMWPE). Recently, Sawatari et al. found that dried gels of LMWPE with molecular weight $<3 \times 10^{5}$ do not form a film. This is one of conclusions for the above question. ${ }^{12}$

The production of high modulus fibers with LMWPE has been developed by Ward et al. ${ }^{13-15}$ and Porter et al. ${ }^{16,17}$ using hydrostatic extrusion. According to the report by Ward et al., ${ }^{13}$ the weight average molecular weight, $\bar{M}_{w}$, was very important in determining the maximum draw ratio which could be obtained. They pointed out that this phenomenon is attributed to the reduction in interlameller tie molecules in the undrawn state with increasing

\footnotetext{
† On leave from Tongyang Nylon Co., Ltd., Hone-Dong Anyang-Si 183, Korea.

* To whom all correspondence should be addressed.
} 
$\bar{M}_{w}$. Furthermore, Porter et al. ${ }^{16}$ pointed out that the Young's modulus versus draw ratio of extruded high density polyethylene depends on the extrusion temperature and pressure condition. Recently, the investigation of high modulus fibers of LMWPE has been developed by melt spinning and high modulus fibers have been produced in a plant level of institute of companies. Unfortunately, the detailed manufacturing has never been opened to the publics.

It is certain that LMWPE cannot still produce fibers whose Young's modulus is higher than $100 \mathrm{GPa}$ but can produce fiber with the Young's modulus of about $70 \mathrm{GPa}$. This is a very important event, since the Young's modulus of $50 \mathrm{GPa}$ is enough to usual applications except special purposes. Such fibers are very important in a commercial base, since the production rate of high modulus fibers from UHMWPE gels is far less than commercially interesting speed because of a drastic increase in viscosity with increasing concentration of solution.

In this paper, we adopt fibers and calender films produced from different LMWPE by some companies in order to study the origin of facile drawability associated with high modulus and high strength. The fibers produced by melt spinning on the basis of Ward's guidance, assure the Young's modulus above 50 $\mathrm{GPa}$, while the Young's modulus of the calender films is less than $20 \mathrm{GPa}$ at $20^{\circ} \mathrm{C}$, although the second order orientation of the $c$-axes and amorphous chain segments of both the specimens are almost equal. In this study, the morphological feature was investigated on a submicroscopic scale by using various techniques such as wide angle $\mathrm{X}$-ray diffraction (WAXD), small angle X-ray scattering (SAXS), small angle light scattering (SALS), scanning electron microscopy (SEM), and differential scanning calorimetry (DSC) to explain the differences in the morphological properties.

\section{EXPERIMENTAL}

Films and fibers of LMWPE were used as specimens in this experiment. In order to prepare the films, a linear polyethylene with a viscosity average molecular weight $\bar{M}_{v}$ of 70,000 was calendered under different friction ratios of the roll at $100-135^{\circ} \mathrm{C}$. The draw ratio of the calender film was determined as the friction ratios of the roll in a company. On the other hand, in accordance with Ward's method used in another company, momofilaments (undrawn fiber) of a linear polyethylene with $\bar{M}_{v}=54,000$ were prepared by melt spinning under the cooling rate of 2 $10^{\circ} \mathrm{C}$ per minute. According to the suggestion of this company, to assure high drawability, the structure produced when the temperature reaches $115^{\circ} \mathrm{C}$ must be preserved by the rapid cooling in the first stage. In order to promote the molecular orientation, the filaments were drawn at temperatures above $70^{\circ} \mathrm{C}$ as the second stage. The detailed know-how for preparing both specimens, however, is kept at each company.

The viscosity average molecular weight $\bar{M}_{v}$ is calculated from the relationship of $[\eta]=K \bar{M}_{v}$ assuming $K=6.2 \times 10^{-4}$ and $a=0.67,{ }^{18}$ in which $[\eta]$ is defined by $\lim _{c \rightarrow 0} \eta_{\mathrm{sp}} / c$ (c, concentration and $\eta_{\mathrm{sp}}$, the specific viscosity).

The crystallinity was calculated from the density which was measured by a pycnometer in chlorobenzene-toluene as a medium, using 1.000 and $0.852 \mathrm{~g} \mathrm{~cm}^{-3}$ as densities of the crystal and amorphous phases. ${ }^{19}$ The melting point was estimated in terms of melting endotherms of DSC curves obtained at a constant rate of $10^{\circ} \mathrm{C} \mathrm{min}{ }^{-1}$. Specimens, weighting in $5 \mathrm{mg}$ each, were placed in a standard aluminium sample pan. Birefringence measurements were made by a polarizing microscope using Berek compensator for measuring the retardation.

The mechanical properties were measured with a tensile tester at room temperature and 
with a viscoelastic spectrometer (VES-F) obtained from Iwamoto Machine Co., Ltd. over the temperature range -150 to $113^{\circ} \mathrm{C}$. The specimens of calender films cut to a length of $60 \mathrm{~mm}$ and the width of $1.5 \mathrm{~mm}$ and the fibers also cut to a length of $60 \mathrm{~mm}$ were clamped over a length of $10 \mathrm{~mm}$ at the end.

The X-ray measurements were carried out by $12 \mathrm{KW}$ ratating anode $\mathrm{X}$-ray generator (RDA-rA). WAXD patterns were obtained with a flat film camera using $\mathrm{Cu}-K_{\alpha}$ radiation monochromator. The exposure for all specimens was $30 \mathrm{~min}$ and $1 \mathrm{~h}$, when the incident beam was directed parallel (through view) and normal to the film surface (end view), respectively. SAXS patterns were obtained with a flat film camera. Each exposure time for a specimen was $100 \mathrm{~h}$. SAXS intensity distribution in the meridional direction was detected with a position sensitive proportional counter (PSPC) at $200 \mathrm{~mA}$ and $40 \mathrm{kV}$. The measurements were carried out by point focus with a three-pin hole collimator system with the counting interval exposure time of $4 \times$ $10^{5} \mathrm{~s}$. The corrected intensity was obtained by substracting the contribution of the background (corresponding - to air scattering) from the total intensity.

Scanning electron microscope and optical micrographs were obtained with a JSM-T300 and a Nikon optical polarizer (XTP-11), respectively.

SALS patterns were obtained with a 3-mW $\mathrm{He}-\mathrm{Ne}$ gas laser as the light source. Diffuse scattering was avoided by sandwiching the specimen between cover glasses with a silicon immersion oil having a similar index.

The orientation of three crystallographic axes was estimated in terms of the second order orientation factor from the X-ray diffraction intensity distribution. The evaluation of the orientation of amorphous chain segments was obtained from birefringence data as estimated by subtraction of crystalline contribution from the total birefringence, assuming simple additivity proposed by Stein and Norris. ${ }^{20}$ In the calculation, the intrinsic birefringences of the crystalline and amorphous phases are estimated as $58.5 \times 10^{-3}$ and $53.0 \times$ $10^{-3}$, respectively, from the three principal refractive indices of crystal of the $n$-paraffin $\left(\mathrm{C}_{36} \mathrm{H}_{74}\right)$ reported by Bunn and de Daubeny. ${ }^{21}$ This calculation is based on the assumption that the principal refractive indices can be estimated by assuming the atomic arrangements within the crystal unit cell and amorphous phase and neglecting the uncertain effects of the internal field.

\section{RESULTS AND DISCUSSION}

Table I shows the characteristics of the calender films with different draw ratios and undrawn and drawn fibers produced by melt spinning. In Table I, the melting point, the volume crystallinity, and the storage modulus of the calender films at $20^{\circ} \mathrm{C}$ increase gradually with increasing draw ratio $\lambda$. The birefringence indicates a drastic increase at smaller draw ratios than $\lambda=3$ and a gradual increase beyond $\lambda=3$. The characteristics of the undrawn fiber are similar to those of the original film $(\lambda=1)$

Table I. Characteristics of calender films with different draw ratios and undrawn and drawn fibers produced by melt spinning

\begin{tabular}{lcccccccccc}
\hline \multicolumn{1}{c}{ Specimen } & \multicolumn{1}{c}{ Calender films } \\
\hline Draw ratio $(\lambda)$ & 1 & 3 & 6 & 8 & 10 & 12 & 14 & Undrawn & Drawn \\
\hline Melting point $/{ }^{\circ} \mathrm{C}$ & 130 & 133 & & & 134.5 & 134.5 & 135 & 134 & 141.5 \\
Crystallinity $/ \%$ & 69.2 & 70.6 & 72.1 & 73.9 & 77.0 & 77.6 & 78.6 & 68.4 & 86.4 \\
Storage modulus at $20^{\circ} \mathrm{C}(\mathrm{GPa})$ & 1.59 & 2.71 & 3.98 & 4.30 & 10.1 & 13.3 & 15.3 & 1.85 & 55.6 \\
Birefringence $\left(\times 10^{-3}\right)$ & -0.53 & 40.5 & 44.8 & 46.6 & 47.9 & 47.9 & 50.3 & 3.36 & 48.0 \\
\hline
\end{tabular}




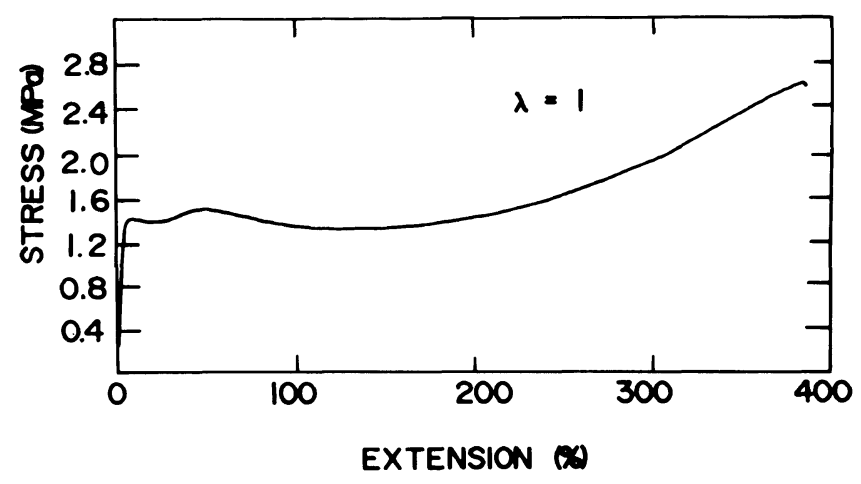

Figure 1. Stress-strain relationship of the original calender film with $\lambda=1$.

but the drawing causes significant changes in the melting point, the crystallinity, and the storage modulus at $20^{\circ} \mathrm{C}$, except the birefringence. Here it is of interest that the storage modulus of the drawn fiber is much higher than that of the drawn film with $\lambda=14$, although the birefringence of the fiber is slightly lower than that of the drawn film. This paper is focused on characteristic differences of both specimens in terms of mechanical and morphological aspects.

Figure 1 shows the nominal stress-strain relationship of the original calender film $(\lambda=1)$ at room temperature and at a cross-head speed of $10 \mathrm{~mm} \mathrm{~min}^{-1}$. The stress decreases with increasing draw ratio and reaches a minimum value around $120 \%$. Such a decrease can be observed when soft polymeric materials are drawn at such a slow strain speed as $10 \mathrm{~mm}$ $\min ^{-1}$. This is attributed to stress-relaxation under elongation. Beyond $120 \%$, however, the stress increases with the draw ratio and the film can be drawn to $400 \%$ of the original length.

Figure 2 shows the nominal stress-strain relationship of the calender films with $\lambda=3$ and 14 measured at the same condition in Figure 1. The tensile strength of the film with $\lambda=14$ is less than $50 \mathrm{MPa}$.

Figure 3 shows the nominal stress-strain relationship of the undrawn and drawn fibers measured at the same conditions in Figures 1
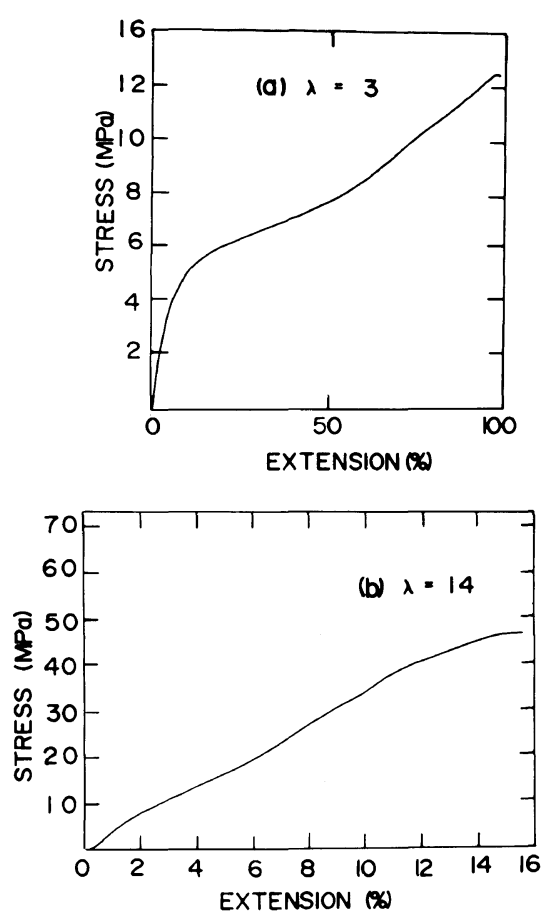

Figure 2. Stress-strain relationship of the calender film with $\lambda=3$ and 14 .

and 2. The formation of a neck was observed under the elongation. This evidently shows that the mono-filament can be stretched up to a remarkably high draw ratio of $\lambda=46$. Such a phenomenon is probably due to the morphological properties of the original (undrawn) specimen which causes a significant crystal transition from a folded to a fibrous 

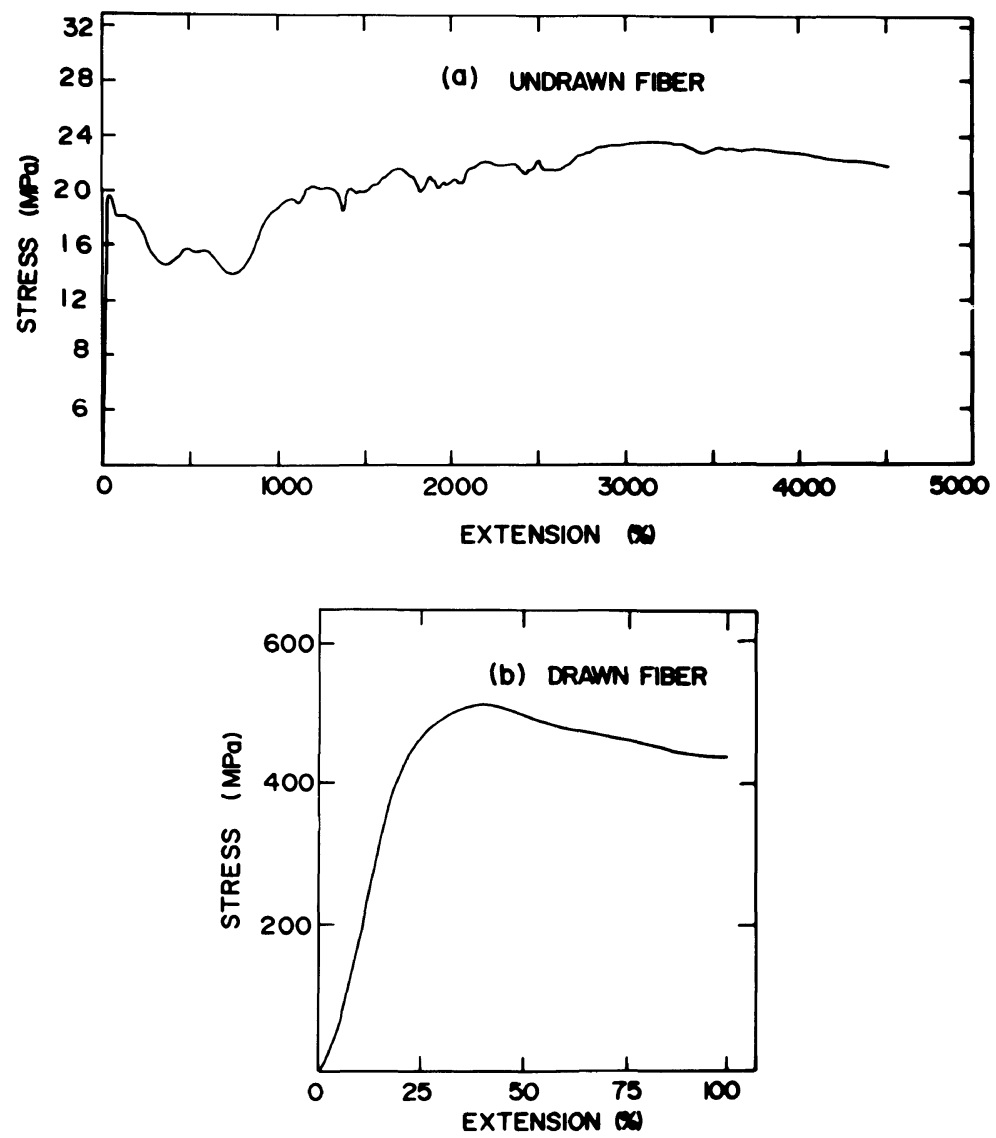

Figure 3. Stress-strain relationship of the undrawn and drawn fibers.

type. This drawability is much higher than that of LMWPE filaments reported by Ward et al. ${ }^{13}$ at room temperature. According to their report, ${ }^{13}$ the maximum draw ratio was less than 13 times for LMWPE with $\bar{M}_{w}=40,000$ and the draw ratio decreased with increasing $\bar{M}_{w}$. The phenomenon of necking was also observed for the fiber used in this experiment under elongation up to $\lambda=40$ in a hot oven in the range $100-130^{\circ} \mathrm{C}$, as has been observed for the drawing of UHMWPE up to $\lambda=50$ at $135^{\circ} \mathrm{C}$. The maximum draw ratio of this fiber was less than 70 times and the corresponding Young's modulus was about $70 \mathrm{GPa}$.

Figure 4 shows the temperature dependence of the complex dynamic tensile moduli of the calender films with the indicated draw ratios.
All the specimens were annealed for $30 \mathrm{~min}$ at $110^{\circ} \mathrm{C}$ prior to the measurements in order to discuss the profiles of the storage and loss moduli in relation to the draw ratio without considering thermal crystallization. The storage modulus $E^{\prime}$ decreases with increasing temperature. The value becomes higher as $\lambda$ increases and this tendency is significant over the whole temperature range. The value of $E^{\prime}$ for the drawn film with $\lambda=14$ was $15.3 \mathrm{GPa}$ at $20^{\circ} \mathrm{C}$ as shown in Table $\mathrm{I}$.

The draw ratio also causes a significant change in the profile of the loss modulus $E^{\prime \prime}$. The relationship exhibits the $\alpha$ and $\gamma$ peaks in order of decreasing temperature. According to studies of viscoelastic properties of crystalline polymers, ${ }^{22.23}$ it is well known that the $\alpha$ 

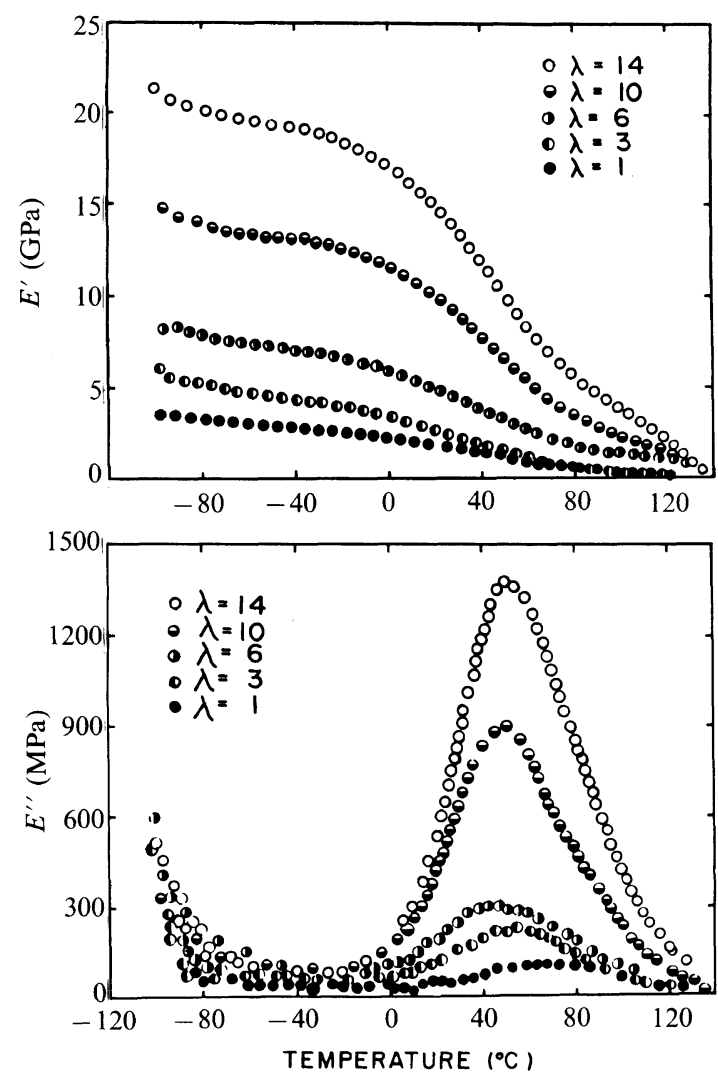

Figure 4. Temperature-dependence of the complex dynamic tensile modulus of the calender films with the indicated draw ratios.

dispersion peak corresponds to the mechanism associated with crystal dispersion which can be classified into two components; the $\alpha_{1}$ mechanism is associated with grain boundary phenomena concerning deformation and/or rotation of crystallites (crystal mosaic block) within a viscous medium, and the $\alpha_{2}$ mechanism with the crystal disordering transition due to the onset of torsional oscillation of polymer chains within the crystal lattice. The peak associated with the $\alpha$ transition appeared around $50^{\circ} \mathrm{C}$, which is independent of draw ratio. The peak position of the $\gamma$ transition appeared at temperatures below $-80^{\circ} \mathrm{C}$. The $\gamma$ transition is usually assigned to the local mode relaxation from twisting of the main chains in the amorphous phase as well as that from
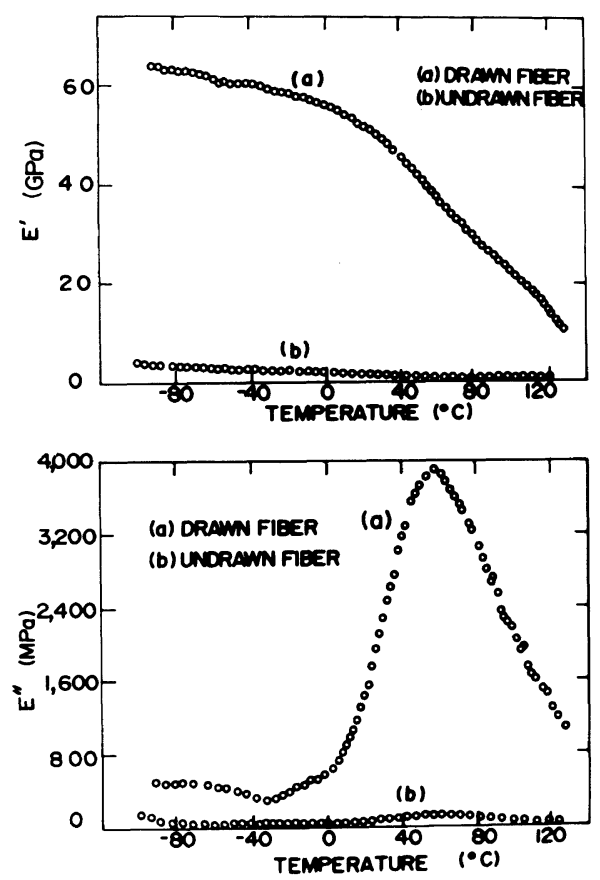

Figure 5. Temperature-dependence of the undrawn and drawn fibers.

lattice defects within the crystal phase.

Figure 5 shows the temperature dependence of the original and drawn fibers. The magnitude of the storage modulus $E^{\prime}$ of the drawn fiber is much higher than that of the drawn film with $\lambda=14$ in Figure 4 over the whole temperature range but the dispersion peak of the $\alpha$ transition appeared around $50^{\circ} \mathrm{C}$ as in the case of the drawn film with $\lambda=14$. The temperature for all the specimens is lower than those of undrawn and drawn UHMWPE gel films $\left(80^{\circ} \mathrm{C}\right){ }^{24,25}$ This indicates that the $\alpha$ dispersion peak is independent of molecular orientational degree and crystallinity but is affected by molecular weight.

Figure 6 shows WAXD pattern (end view) of the original (undrawn) calender film, taken with the incident beam directed parallel to the mechine direction. The photograph exhibits cylindrical rings indicating the random orientation of crystallites around the machine direction. 


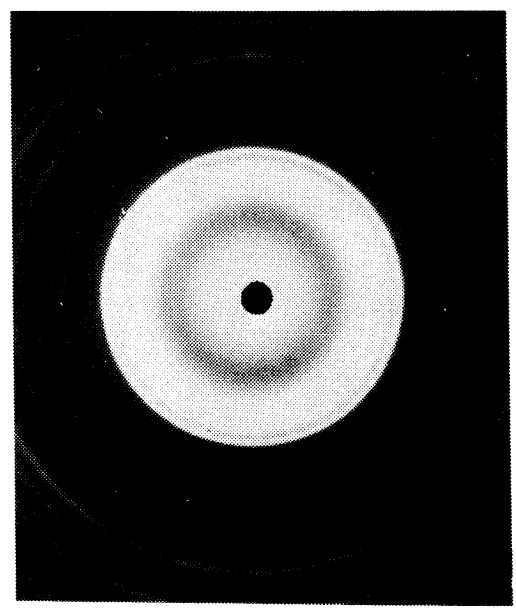

Undrawn film (end view)

Figure 6. WAXD pattern (end view) from the original calender film with $\lambda=1$.

Figure 7 shows WAXD patterns (through view) of the film with various draw ratio $\lambda$. It is seen in Photo. (a) in Figure 7 that the undrawn film exhibits preferential orientation of the $a$ axes with respect to the machine direction. Such behavior has been observed for calender films prepared by LMWPE with molecular weights below $150,000 .^{26}$ The calender films with molecular weight above 300,000 were reported to take the preferential orientation of the $c$-axes. ${ }^{26}$

Figure 8 shows the SAXS patterns of calender films with $\lambda=1$ and 14 . The pattern of the original film $(\lambda=1)$ shows up to the second order scattering maxima whose lobes are extended in the meridional direction. The pattern from the drawn film with $\lambda=14$ shows distinct scattering maxima whose lobes are extended in the horizontal direction. The profiles of the two SAXS patterns have been observed for calender films. ${ }^{24}$ Observation up to the second order maxima revealed that the undrawn film is composed of crystal lamellae oriented with their large flat faces perpendicular to the machine direction and such a orientational mode of lamellae is preserved during the drawing process up to $\lambda=14$. The SAXS pattern (b) in

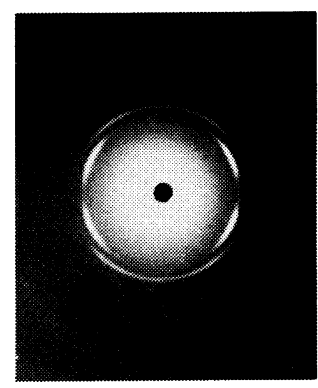

(a) $\lambda=1.0$

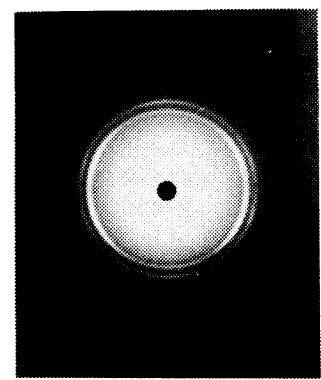

(b) $\lambda=1.2$

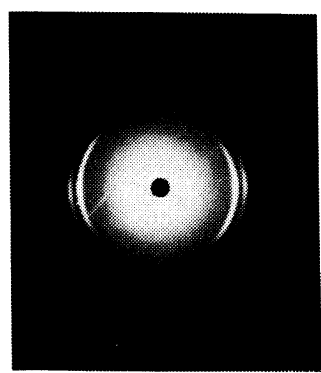

(c) $\lambda=3.0$

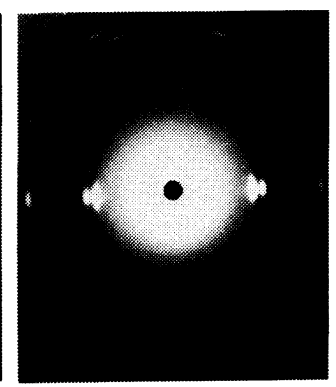

(d) $\lambda=6.0$

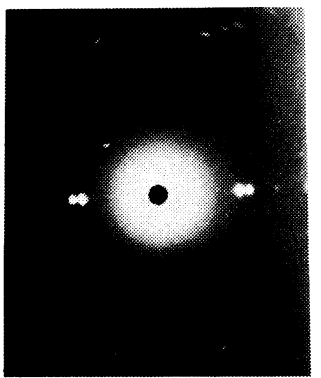

(e) $\lambda=10.0$

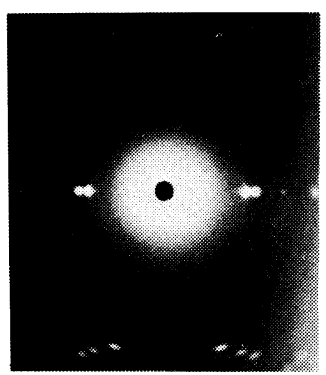

(f) $\lambda=14.0$
Figure 7. WAXD patterns (through view) from the calender films as a function of draw ratio $\lambda$.

Figure 8 and the WAXD pattern $(f)$ in Figure 7 together indicate that within the lamellar crystals of the drawn film with $\lambda=14$, the $c$ axes are oriented perpendicular to the large faces, which shall be discussed later in detail.

Figure 9 shows $H_{\mathrm{v}}$ SALS patterns as a function of draw ratio $\lambda$. The original film $(\lambda=$ 1) shows a indistinct pattern whose intensity distribution is independent of the azimuthal angle. This is a typical scattering from a system having a random array of crystallites of which size is small compared with the wavelength of an incident beam. Elongation up to $\lambda=3$ 


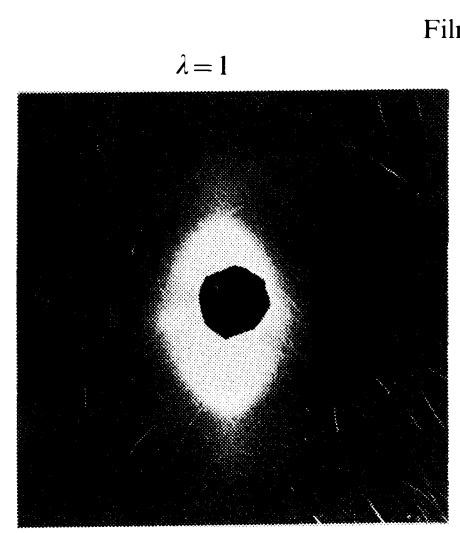

(a)

Film

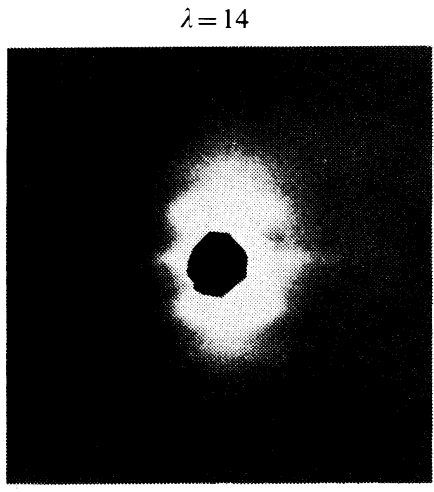

(b)

Figure 8. SAXS patterns from the calender films with $\lambda=1$ and 14 .

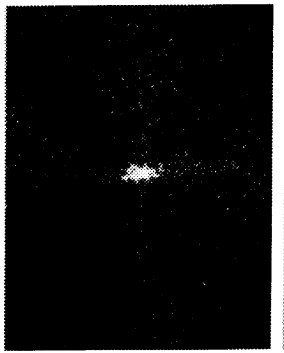

(a) $\lambda=1$

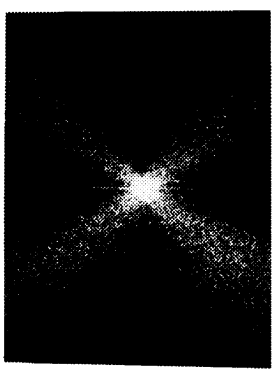

(b) $\lambda=3$

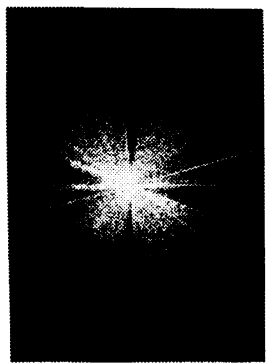

(c) $\lambda=6$

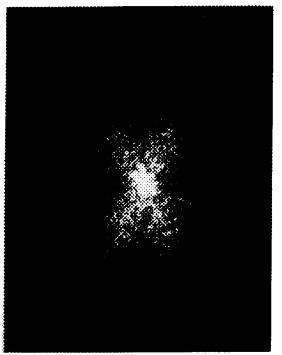

(d) $\lambda=10$

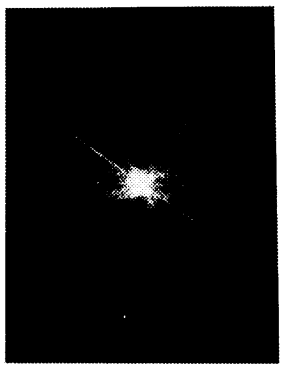

(e) $\lambda=14$
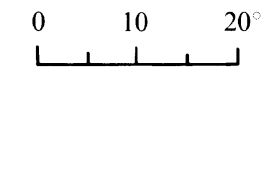

Figure 9. $H_{\mathrm{v}}$ SALS patterns from the calender films as a function of draw ratio $\lambda$. causes development of the X-type pattern. The intensity continuously decreases with increasing scattering angle. Such a pattern reveals typical scattering from rods, the optical axes being parallel or perpendicular to the rod axis. Interestingly, the pattern shows maximum intensity at odd multiples of $45^{\circ}$. Such a profile has been observed for an undrawn film, indicating random orientation of rods. As shown in Figure 7, however, the crystallites take preferential orientation with respect to the machine direction. This indicates that the rods orient at $\pm 45^{\circ}$ with respect to the machine direction and the $c$-axes coresponding to the optical axes orient predominantly in the machine direction. Such a noble orientation discerned from the fact that the notch of the four lobes is more clearly defined than those observed for undrawn films generally.

With increasing $\lambda$ up to 10 , the scattering lobes are extended in the meridional direction. Such patterns have been observed under the oriented crystallization process for UHMWPE films in the range $\lambda=15-20{ }^{8}$ This indicates the orientation of rods perpendicular to the stretching direction. Finally, the pattern at $\lambda=14$ displays indistinct small lobes indicating the disappearance of rods and/or the almost perfect orientation of the optical axes within the rods in the stretching direction. 


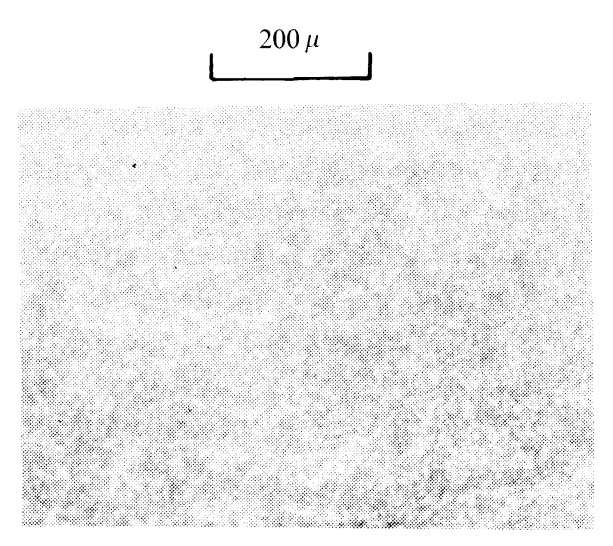

(a) $\lambda=1$

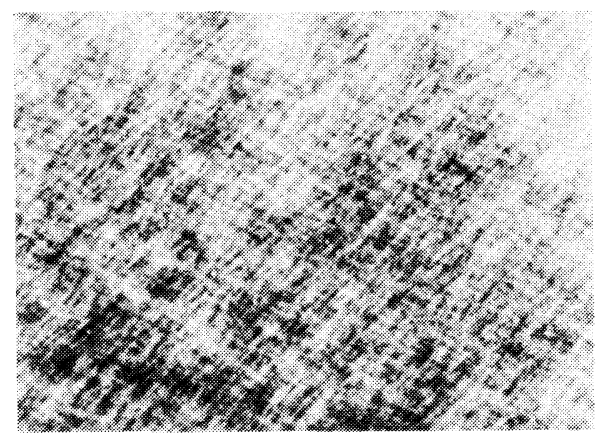

(b) $\lambda=3$

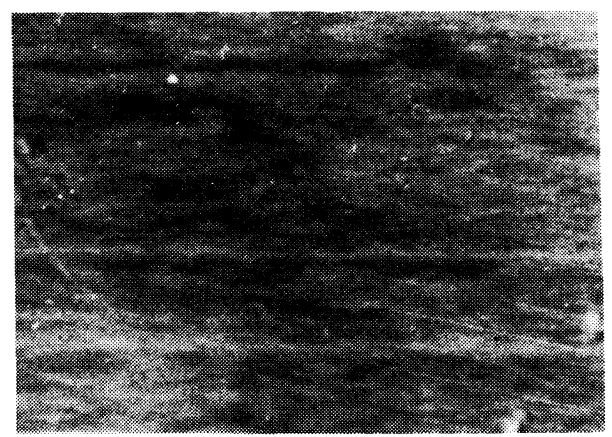

(c) $\lambda=10$

Figure 10. Optical micrographs (cross-polarized) of the calender films at various draw ratios $\lambda$.

In the polarizing microscope, the development of the fibrous texture could be readily followed. Figure 10 shows the change in the appearance of the specimen with stretching. Under the scale of magnification, it is very

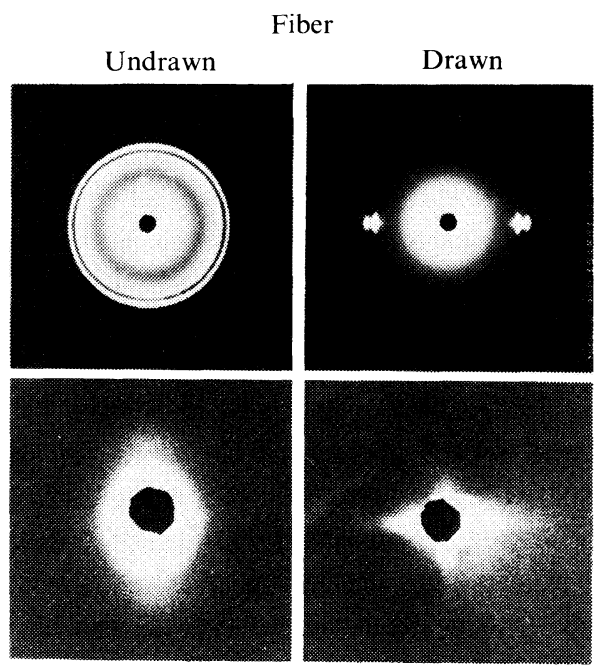

Figure 11. WAXD and SAXS patterns (through view) from the undrawn and drawn fibers.

difficult to recognize morphological features, namely, rods or spherulites within the original films. At $\lambda=3$, close observation reveals that the textures are composed of a stack of oriented lamellae. These units are oriented at a particular angle of $\pm 45^{\circ}$ with respect to the machine direction to form a network structure. This observation is in good agreement with the SALS pattern of the film with $\lambda=3$ in Figure 9. This photographs and the SALS pattern together indicate that the network structure observed on the film surface remains in the interior of the specimen. Beyond $\lambda=10$, the width of rods decreased and finally the rodlike textures seemed to be disruptively deformed into fine fibrils. The fibrils were also highly oriented perpendicular to the machine direction. As an example, the photograph at $\lambda=10$ is listed.

Next, in order to understand the difference of the morphological properties between the films and the fibers, the morphological properties of the fibers is discussed based on WAXD SAXS, and scanning electron micrographs.

Figure 11 shows WAXD and SAXS patterns (through view) of. undrawn and drawn fibers. The $a$-axes of the undrawn fiber orient 
predominantly with respect to the machine direction but the degree of orientation is not so great as that of the original calender film shown in Figure 7. The angular spreads of the strong (110) and (200) reflections of the drawn fiber indicates an increase in the preferential orientational degree of the $c$-axes with respect to the stretching direction. The orientational degree, however, is less pronounced than that of the drawn film with $\lambda=14$ in Figure 7 .

In the SAXS pattern of the undrawn film, the scattering maxima are indistinct and especially the second order maximum becomes very weak. The scattering maxima are displaced toward the center of the pattern by the drawing but this tendency is very difficult to discern because of the superposition of the void scattering. Detailed analysis of SAXS intensity distribution is discussed using a PSPC system later in this paper.

Figure 12 shows the change in the appearance of the undrawn and drawn fibers under SEM. The array of fiber structures can be observed even in the undrawn fiber and elongation causes development of this array. More quantitative evaluation for the crystalline orientation and lamellar orientation were investigated in terms of the second order orientation factors of the three principal crystallographic axes and the amorphous chain segments as well as the SAXS intensity distribution in the meridional direction by a PSPS system.

Table II shows the orientation factors of the $a, b$, and $c$ axes corresponding to the factors of the reciprocal lattice vectors of the (200), (020), and (002) planes for the films and fibers. In order to check the validity of the values of the $b$ and $c$ axes, the orientation factors of the $b$ and $c$ axes were estimated from the factors concerning the (110) and (200) planes by Wilchinsky's equation. ${ }^{27}$ The values obtained by both the methods were almost same. In the original calender film $(\lambda=1)$, the $a$ axes take preferential oreintation with respect to the machine direction, while the $c$-axes and the

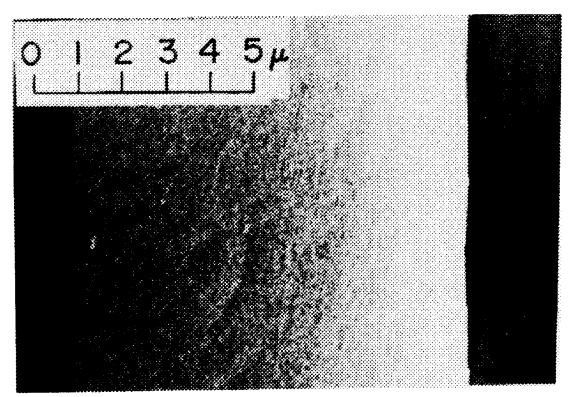

(a) Undrawn fiber

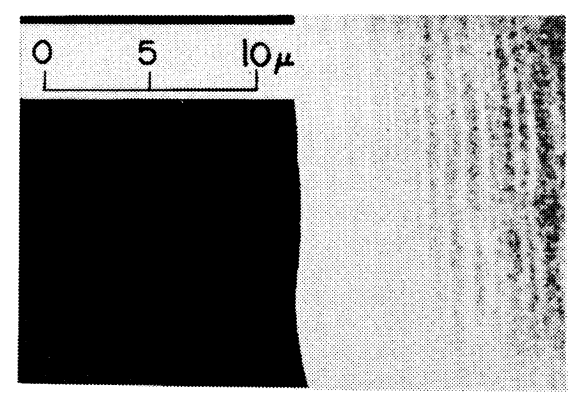

(b) Drawn fiber

Figure 12. Scanning electron micrographs of undrawn and drawn fibers.

Table II. The second order orientation factors of three principal crystallographic axes and amorphous chain segments of calender films with different draw ratios and undrawn and drawn fibers by melt spinning

\begin{tabular}{lrrrrrrrrr}
\hline \multicolumn{1}{c}{ Specimen } & \multicolumn{1}{c}{ Calender films } & & \multicolumn{2}{c}{ Fibers } \\
\hline Draw ratio $(\lambda)$ & 1 & 3 & 6 & 8 & 12 & 14 & Undrawn & Drawn \\
\hline a-axis & 0.4268 & -0.3896 & -0.4348 & -0.4490 & -0.4589 & -0.4763 & 0.2449 & -0.4099 \\
$b$-axis & -0.4142 & -0.3700 & -0.3907 & -0.4115 & -0.4147 & -0.4528 & -0.3564 & -0.4344 \\
$c$-axis & -0.0126 & 0.7596 & 0.8256 & 0.8605 & 0.8763 & 0.9291 & 0.1116 & 0.8443 \\
Amorphous chain segments & 0.0029 & 0.6367 & 0.7300 & 0.7431 & 0.7477 & 0.7512 & -0.0479 & 0.8235 \\
\hline
\end{tabular}




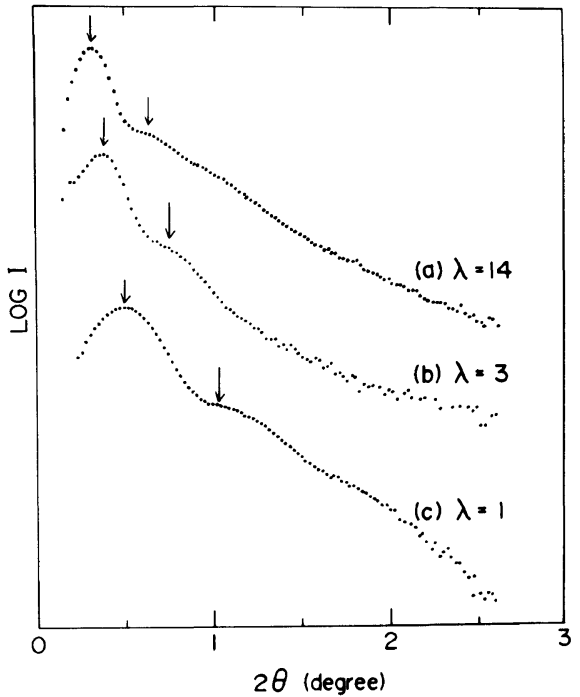

Figure 13. SAXS intensity distributions as a function of twice the Bragg angle $(2 \theta)$ in the meridional direction for the calender films with $\lambda=1,3$, and 14

amorphous chain segments take almost random orientation. With increasing draw ratio, the $c$ axes and amorphous chain segments orient predominantly in the machine direction and the orientational degrees of the $c$-axes is more pronounced than that of the amorphous chain segments.

For the undrawn fiber, the $a$-axes take the preferential orientation with respect to the machine direction and the $b$ axes orient almost perpendicular. The $c$-axes and the amorphous chain segments of the drawn fiber orient preferentially in the stretching direction. In comparison with the values of the orientation factors in Table II, the orientational degree of the $c$-axes of the drawn fiber is less pronounced than that of the drawn film with $\lambda=14$, while the degree of the amorphous chain segments is slightly higher than that of the drawn film.

Figure 13 shows the SAXS intensity distribution in the meridional direction for the calender films. For all specimens, the scattered intensity shows up to the second order maxima whose magnitude decrease with increasing scattering angle. It is of interest to note the fact

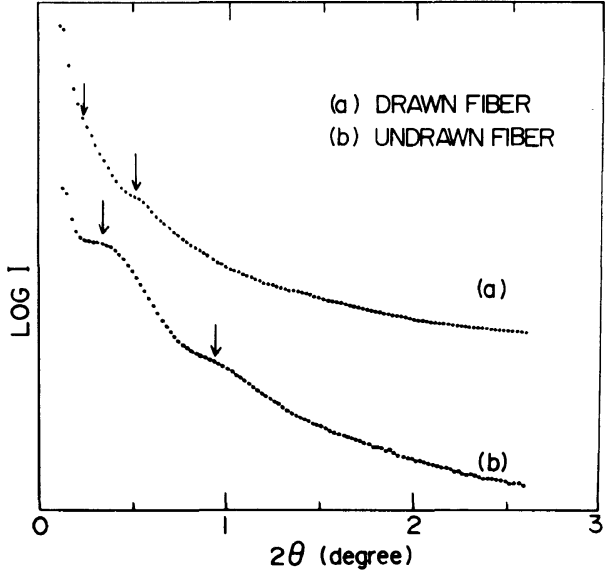

Figure 14. SAXS intensity distributions as a function of twice the Bragg angle $(2 \theta)$ in the meridional direction for the undrawn and drawn fibers.

that the first order maximum becomes distinct and slightly shifts to the center of the scattering angle as the draw ratio increases. The identity periods of the films with $\lambda=14,3$, and 1 are calculated to be about 280,230 , and $180 \AA$, respectively. These values are calculated by the Bragg's eauation. Incidentally, similar values are also obtained by the second order scattering maximum. The increase in the identity period with draw ratio means that there exist crystal lamellae and crystal lamellae undergo large changes in the orientation, lamellar thickness, and identity period as a result of the recrystallization associated with the refolding to a large fold period under elongation process up to $\lambda=14$.

Figure 14 shows SAXS intensity distributions in the meridional direction for the fibers. For the undrawn and drawn fibers, the scattered intensity for the undrawn fiber shows the second order maxima with small peaks. These profiles indicate the large fluctuation of crystal lamellar thickness as well as that of crystal orientation. The identity periods of the undrawn and drawn fibers are about 225 and $365 \AA$, respectively. These values are estimated by using the first order scattering maximum but the values estimated from the second order 


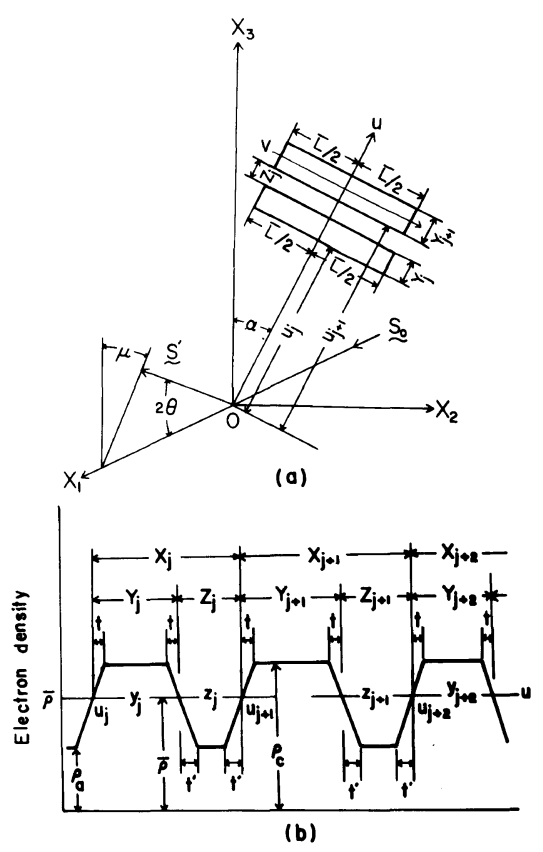

Figure 15. (a) A two-dimensional model used for the theoretical analysis of the SAXS intensity distribution. (b) Trapezium density profile, in which the density variation between the two phases is specified by positive and negative deviations from the average of the system.

maximum are different from the above values. The profile of the first order peak is duller than that of the films shown in Figure 13. This indicates that the fluctuations of lamellar orientation and lamellar thickness of fibers are much larger than those of the films.

In order to clarify the differences quantitatively, SAXS intensity distribution were computed in terms of a two-dimensional model of periodic crystalline-amorphous structures in Figure 15. This treatment was made on the basis of the concept that scattering is caused by positive and negative derivations from the density of the sample. ${ }^{28.29}$ A twodimensional model is not so realistic as a threedimensional one. In the present paper, however, a two-dimensional model is proposed, since a three-dimensional model is too complicated to represent the scattered intensity by introducing the average density. A detailed explanation for a three-dimensional model will

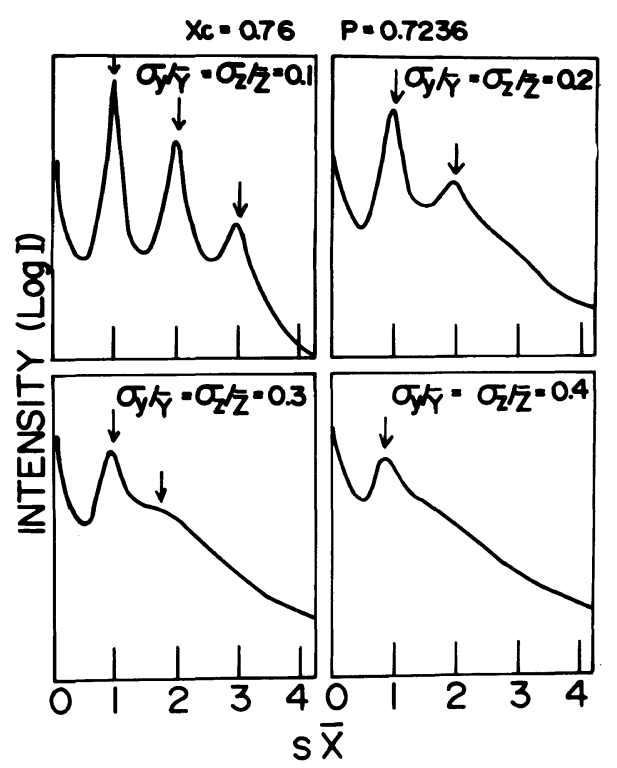

Figure 16. The profile of $\log I$ as functions of $\sigma_{y} / \bar{Y}$ and $\sigma_{z} / \bar{Z}$ at $X_{\mathrm{c}}=0.76$.

be discussed elsewhere. ${ }^{30}$ The numerical calculation in this paper was carried out using a two-dimensional system. The notation of all parameters in the previous paper ${ }^{29}$ is preserved in this paper. In numerical calculation, the parameter $\sigma_{\alpha}$ denoting the orientational degree of lamellae is fixed to be 5 and the average number of lamellae is assumed to be 10 .

Figure 16 shows the results calculated as a function of the fluctuation in the thicknesses of the crystalline and amorphous regions, that is, $\sigma_{y} / \bar{Y}$ and $\sigma_{z} / \bar{Z}$. The volume crystallinity $X_{\mathrm{c}}$ is fixed to be $0.76(76 \%)$, corresponding to the crystallinity of the film with $\lambda=14$. The lengths $t$ and $t^{\prime}$ of the transition zones are normalized by $\bar{X}$ and $t / X$ and $t^{\prime} / \bar{X}$ are fixed to be 0.0163 and 0.0532 , respectively, authomatically when $p=(\bar{Y} / \bar{X})$ is 0.7236 . The height of the maxima of logarithmic term of scattered intensity $(\log I)$ decreases considerably with increasing $s \bar{X}$ and becomes a smooth monotonically decreasing line with further increases of $s \bar{X}$. Namely, higher order maxima at $s \bar{X}>4$ are smeared out completely. When four curves are compared, it is seen that the increases of $\sigma_{y} / \bar{Y}$ 


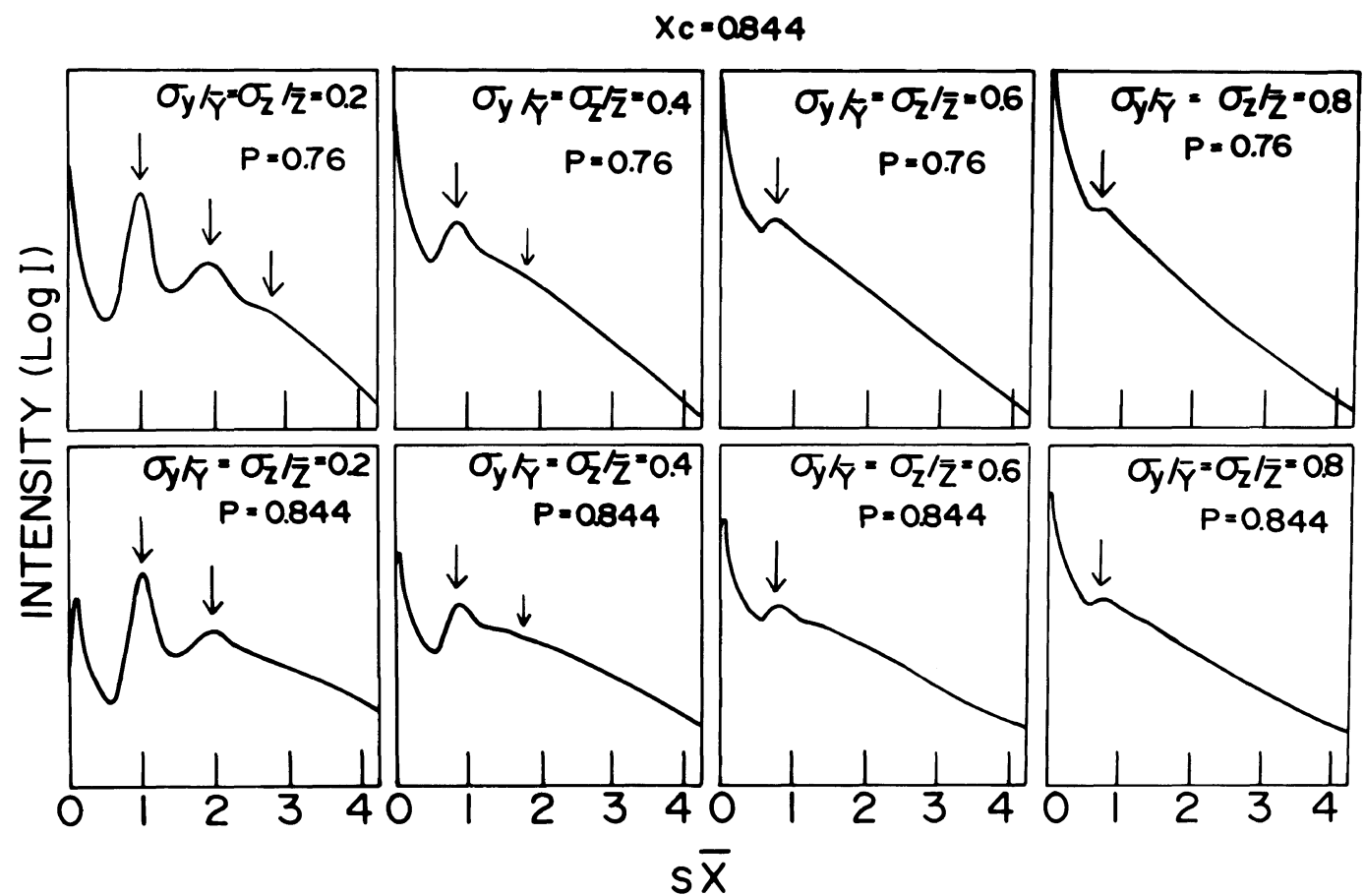

Figure 17. The profile of $\log I$ as functions of $\sigma_{y} / Y$ and $\sigma_{z} / Z$ at $X_{\mathrm{c}}=0.844$, in which the upper and lower curves are calculated at $p=0.76$ and 0.844 , respectively.

and $\sigma_{z} / \bar{Z}$ make the peak broad.

Figure 17 shows the results calculated as functions of $\sigma_{y} / \bar{Y}$ and $\sigma_{z} / \bar{Z}$ at $X_{\mathrm{c}}=0.844$. The upper curves were calculated at $t / \bar{X}=0.01905$ and $t^{\prime} / \bar{X}=0.1153$, while the lower curves at $t / \bar{X}=t^{\prime} / X^{\prime}=0$. Comparing the upper and lower curves, it is evident that the introduction of the transition zones promotes a rapid decrease of the scattered intensity with increasing $s \bar{X}$. Furthermore, the profile of the intensity around $s \bar{X}=0$ indicates that the existence of the transition zones provides a sharp distribution of the scattered intensity around $s \bar{X}=0$ but in the absence of the transition zone, the intensity decreases around $s \bar{X}=0$. This difference cannot be observed experimentally, since such beams around $s \bar{X}=0$ are eliminated by a lead stopper. The increase in the fluctuation of the thicknesses of the crystalline and amorphous regions causes derivation from the Bragg's equation for higher order scattering maxima. The maximum peak of the second order shifts from $s \bar{X}=2$ to the center considerably. Actually, when the identity period of the fibers with the large fluctuation was calculated from the intensity distribution in Figure 14, the value calculated from the first order maximum is different from that calculated from the second one. Comparison between the observed intensity distributions in Figures 13 and 14 and the calculated ones in Figures 16 and 17 indicates that the fluctuations of the thicknesses of crystalline and amorphous regions for the drawn fibers are much larger than those for the calender film with $\lambda=14$.

Here it may be noted that long periods have never been observed for native cellulose (for example ramie fiber) with a typical fringed micell type by SAXS measurements, while scattered intensity for an undrawn dry gel film of UHMWPE showed up to the fourth order 


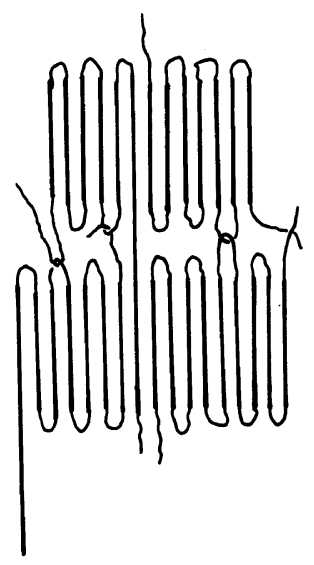

(a)

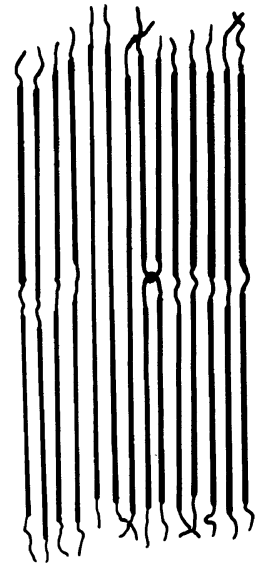

(b)
Figure 18. Schematic diagrams of the crystal orientational behavior of the calender film (model (a)) and drawn fiber (model (b)).

maxima, when an incident beam was directed parallel to the film surface (end view). ${ }^{12}$ This indicates that the fluctuations of the thicknesses of crystalline and amorphous regions taking a fringed type are much larger than those taking a folded type. If this is the case, it may be expected that the crystallites of the calender film with $\lambda=14$ is a folded type and therefore the preferential orientation of the $c$ axes is due to the rotation of crystallites leading to taut tie molecules as shown in Figure 18(a). In contrast, the crystallites of the drawn fiber is thought to be a fibrous type due to a significant crystal transformation as shown in Figure 18(b). This supports the differences of the nominal stress-strain relationship in Figures $1-3$.

Differences of the deformation mechanism between calender film and fiber by melt spinning is thought to be due to a suitable level of entanglement mesh that effectively transmits the drawing force in the original film and fiber. In summary, it is expected that the original melt spun fiber produced by Ward's guidance has a suitable level of entanglement mesh like gel films prepared from solutions with suitable concentrations.

\section{CONCLUSION}

Deformation mechanisms of the calender films and melt spun fibers produced by Ward's method were studied in terms of morphological aspects. Both specimens were produced by LMWPE whose molecular weights are 70,000 and 54,000, respectively. The Young's modulus of the calender film with a maximum draw ratio of $\lambda=14$ is only $15.3 \mathrm{GPa}$, while the modulus of the drawn fiber is $55.6 \mathrm{GPa}$, although the orientation factor of the $c$-axes of the calender film is slightly higher than that of the fiber. This phenomenon is due to the fact that the preferential orientation of the $c$-axes of the calender film is associated with the rotation of crystallites leading to the tension of tie molecules, while that of the fiber is associated with the crystal transition from a folded to fibrous type.

\section{REFERENCES}

1. J. Smook, J. C. Torfs, P. F. van Hulten, and A. J. Pennings, Polym. Bull., 2, 293 (1980).

2. P. J. Barham and A. Keller, J. Mater. Sci., 15, 2229 (1980).

3. T. Kanamoto, A. Tsuruta, K. Tanaka, M. Takeda, and R. S. Porter, Polym. J., 15, 327 (1983).

4. K. Furuhata, T. Yokokawa, and K. Miyasaka, J. Polym. Sci., Polym. Phys. Ed., 22, 133 (1984).

5. P. Smith and P. J. Lemstra, J. Mater. Sci., 15, 505 (1980).

6. P. Smith, P. J. Lemstra, and H. C. Booij, J. Polym. Sci., Polym. Phys. Ed., 19, 877 (1981).

7. P. Smith, P. J. Lemstra, J. P. L. Pippers, and A. M. Kiel, Colloid Polym. Sci, 258, 1070 (1981).

8. M. Matsuo, K. Inoue, and N. Abumiya, Sen- $i-$ Gakkaishi, 40, 275 (1984).

9. M. Matsuo and C. Sawatari, Macromolecules, 19, 2036 (1986).

10. M. Matsuo, C. Sawatari, M. Iida, and M. Yoneda, Polym. J., 17, 1197 (1985).

11. T. Kanamoto, A. Tsuruta, K. Tanaka, M. Takada, and R. S. Porter, Macromolecules, 21, 470 (1988).

12. C. Sawatari, T. Okumura, and M. Matsuo, Polym. J., 18, 741 (1986).

13. J. M. Andrews and I. M. Ward, J. Mater. Sci., 5, 411 (1970).

14. J. Clements, G. Capaccio, and I. M. Ward, J. Polym. Sci., Polym. Phys. Ed., 17, 693 (1979).

15. J. Clements, R. Jackeways, and I. M. Ward, Polymer, 19, 639 (1978). 
16. A. E. Zachariades, W. T. Mead, and R. S. Porter, "Ultra-High Modulus Polymers," A. Ciferri and I. M. Ward, Ed., Applied Science Publishers, London, 1979, Chapter 2, p 77.

17. S. Kojima and R. S. Porter, J. Polym. Sci., Polym. Phys. Ed., 16, 1729 (1978).

18. R. Chiang, J. Phys. Chem., 19, 1645 (1965).

19. C. W. Bunn, Trans. Faraday Soc., 35, 482 (1939).

20. R. S. Stein and F. H. Norris, J. Polym. Sci., 21, 381 (1956).

21. C. W. Bunn and R. de Daubeny, Trans. Faraday Soc., 50, 1173 (1954).

22. M. Takayanagi, Pure Appl. Chem., 15, 555 (1967).

23. J. C. Hoffman, C. Williams, and E. Passaglia, $J$.
Polym. Sci., C, 14, 173 (1966).

24. M. Matsuo, C. Sawatari, and T. Ohhata, Macromolecules, 21, 1317 (1988).

25. M. Matsuo and C. Sawatari, Macromolecules, 21, 1653 (1988).

26. T. Hashimoto, K. Nagatoshi, A. Todo, and $\mathrm{H}$. Kawai, Polymer, 17, 1063 (1976).

27. Z. W. Wilchinsky, J. Appl. Phys., 31, 1969 (1960).

28. M. Matsuo, C. Sawatari, M. Tsuji, and R. S. J. Manley, J. Chem. Soc., Faraday Trans. 2, 79, 1593 (1983).

29. M. Matsuo and C. Kitayama, Polym. J., 17, 479 (1985).

30. M. Matsuo, to be submitted. 